Research Article

\title{
Cervus elaphus Foraging Impacts on Plants and Soils at an Ungrazed Desert Grass/Shrubland in Northwestern New Mexico, USA
}

\author{
Louis C. Bender' ${ }^{1}$ and Jessica R. Piasecke ${ }^{2}$ \\ ${ }^{1}$ Extension Animal and Natural Resources, P.O. Box 30003, MSC 3AE, New Mexico State University, Las Cruces, NM 88003, USA \\ ${ }^{2}$ Department of Fisheries and Wildlife, P.O. Box 30003, MSC 4901, New Mexico State University, Las Cruces, NM 88003, USA
}

Correspondence should be addressed to Louis C. Bender; lbender@nmsu.edu

Received 26 June 2015; Accepted 20 August 2015

Academic Editor: Béla Tóthmérész

Copyright (C) 2015 L. C. Bender and J. R. Piasecke. This is an open access article distributed under the Creative Commons Attribution License, which permits unrestricted use, distribution, and reproduction in any medium, provided the original work is properly cited.

\begin{abstract}
We evaluated Cervus elaphus herbivory and trampling impacts on plants and soils on Chaco Culture National Historical Park (Chaco), a desert grass/shrubland in northwestern New Mexico, USA, most (63\%) of which has been protected from grazing by domestic livestock since 1948. We conducted grazing, browse, and water infiltration surveys in areas which received different amounts of C. elaphus use (use and control), 2004-2007. Browse utilization was $<32 \%$ on monitored species and Odocoileus hemionus use accounted for the majority of browsing. Live plant cover was greater on areas receiving more C. elaphus use, and no grass species were used above recommended levels. Stubble heights of Bouteloua spp. were positively related to relative C. elaphus use on some areas, suggesting possible stimulation of grassland productivity by C. elaphus grazing. Water infiltration rates either did not differ among use or control sites or were faster in use sites, indicating no impacts of C. elaphus use on soil compaction. At current C. elaphus densities $\left(0.2-0.4 / \mathrm{km}^{2}\right)$, negative impacts to plants and soils were not seen on Chaco, and some evidence suggests that light grazing is optimizing desert grasslands of Chaco.
\end{abstract}

\section{Introduction}

Large herbivores such as Cervus elaphus can impact the structure, function, and composition of plant communities through selective feeding [1-7]. Ungulates can affect availability of nitrogen, amount of bare soil, net primary productivity, soil moisture, and many other variables relevant to plant communities [8-10]. These changes can be positive [11, 12] or negative, and this distinction in part depends on intensity of use and specific characteristics of the plant community [13-16]. For example, positive stimulation of productivity in plant communities due to herbivory (i.e., herbivore optimization [12]) has been most commonly associated with mesic grasslands [11, 12, 17], whereas the most significant negative changes in vegetation communities attributed to herbivory are generally associated with woody plants $[1,18,19]$.

Controversy regarding C. elaphus herbivory involves negative effects on recruitment of preferred browse species (such as Salix spp. and Populus tremuloides $[1,18,19]$ ) and on production and diversity of grasslands [7]. Effects such as these make C. elaphus foraging a contentious issue in wildlands management, particularly in protected habitats such as national parks where populations cannot usually be limited by hunting $[20,21]$. Most $\left(86 \mathrm{~km}^{2}\right.$ or $\left.63 \%\right)$ of Chaco Culture National Historical Park (Chaco) in northwestern New Mexico, USA, has been protected from domestic livestock grazing since 1948 , with the remainder $\left(50 \mathrm{~km}^{2}\right.$ or $\left.37 \%\right)$ being protected since 1995 [22, 23]. Furthermore, no wild large grazer is thought to have occurred in Chaco historically, although Odocoileus hemionus, which are mainly browsers, have. Consequently, Chaco is one of the largest exclosures from domestic grazers in the North American west [23] and as such is a valuable baseline reference area regarding the potential condition of southwestern desert grass/shrublands in the absence of grazing. 


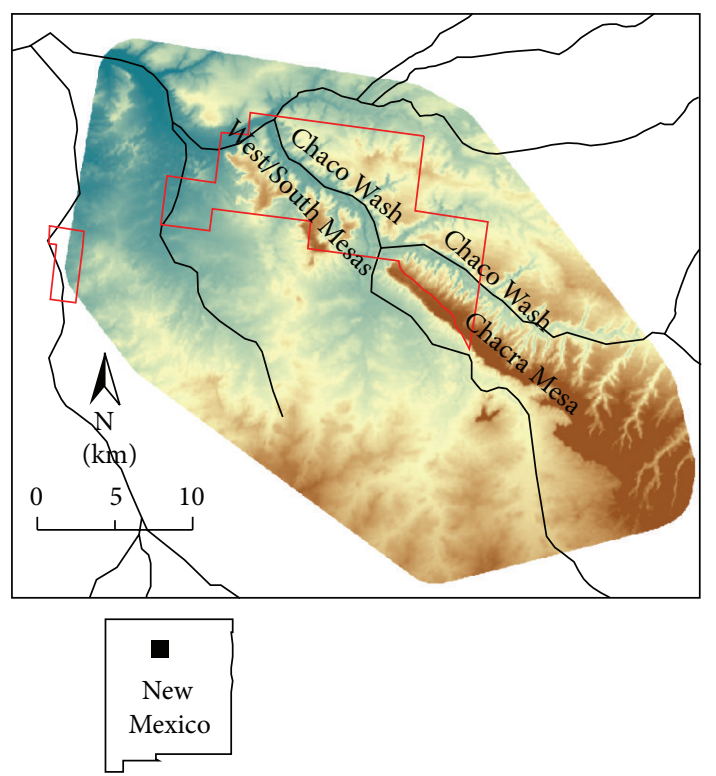

— Streams

— Park boundary

FIgURE 1: Location and topographic relief of Cervus elaphus use area in northwestern New Mexico, USA. Location of Chaco Culture National Historical Park is outlined.

Chaco was colonized by approximately 20 C. elaphus around January 2000, and this herd subsequently increased to $\geq 53$ individuals by 2007 [24]. There is no evidence that $C$. elaphus occurred in Chaco historically, and managers were concerned over the potential impact of C. elaphus on National Park resources, especially riparian woody communities and the relatively pristine ungrazed grasslands. Consequently, our goal was to compare condition and use of riparian and grassland sites in areas receiving relatively heavy and light use by $C$. elaphus in Chaco and to develop a baseline reference for future evaluations if the C. elaphus population continues to increase. Our objectives included (1) determining levels of browsing of key woody plants; (2) determining plant community composition; (3) quantifying degree of grazing on herbaceous communities; (4) determining trends in key noxious plants; and (5) determining effects of C. elaphus trampling on water infiltration.

\section{Methods}

2.1. Study Area. Chaco Culture National Historical Park covers approximately $136 \mathrm{~km}^{2}$ in northwestern New Mexico, USA, centered at approximately $36^{\circ} 00^{\prime} \mathrm{N}, 108^{\circ} 00^{\prime} \mathrm{W}$ (Figure 1). Elevations ranged from 1,670 to $2,079 \mathrm{~m}$, and topography is characterized by rolling plains and mesas interspersed with steep canyons. Average high temperature in July was $32^{\circ} \mathrm{C}$ and average low temperature in January and December was $-11^{\circ} \mathrm{C}$. Average annual precipitation was $23 \mathrm{~cm}$, with $52 \%$ falling in July-October, and average annual snowfall was $37 \mathrm{~cm}$. Chaco Wash, an intermittent stream, bisects the park.
The dominant plant community on mesas consisted of Atriplex canescens, Ericameria nauseosa, Cercocarpus intricatus, Krascheninnikovia lanata, Pleuraphis jamesii, Bouteloua gracilis, Achnatherum hymenoides, and Artemisia bigelovii, with scattered Pinus edulis and Juniperus monosperma. Characteristic vegetation of ravines descending from mesas included Atriplex obovata, Pleuraphis jamesii, Bouteloua gracilis, Bouteloua eriopoda, Sporobolus airoides, Artemisia bigelovii, Krascheninnikovia lanata, Ephedra viridis, and Juniperus monosperma. Vegetation in large washes included Populus spp., Tamarix ramosissima, Atriplex canescens, Pleuraphis jamesii, Sporobolus giganteus, and Sporobolus cryptandrus. Only approximately $15 \%$ of the type of ecosystem represented by Chaco remains intact in North America.

2.2. Browsing Surveys. We conducted browse surveys in late March or early April, 2004-2007, prior to leaf-out or flowering of woody browse species. We established 20 sites each in areas frequented by C. elaphus (use areas) and those not used or used less frequently (control sites) based on distribution of C. elaphus encountered during surveys and movements of radio-collared C. elaphus [24]. The control area included the western portion of Chaco Wash, and the use area included the eastern section of Chaco Wash and draws descending from Chacra Mesa. In both the use and control areas, we randomly placed 10 transects along Chaco Wash, spaced $0.5 \mathrm{~km}$ apart, and 10 in 10 different side canyons. In the use area, all side canyons were off the north side of Chacra Mesa and in the control area off the north side of South and West Mesas. Pseudoreplication was necessary in Chaco Wash areas because of lack of appropriate replication areas (i.e., Chaco Wash was the only deciduous riparian community present).

We used the nearest key browse species method [26] to assess levels of browsing. We sampled the nearest successive shrub in the forward-facing $180^{\circ}$ (i.e., up side canyons or down Chaco Wash [26]), until a maximum of 50 live shrubs $\geq 0.25 \mathrm{~m}$ tall were examined per transect. Occasionally, minimum distances (3-5 m) between subsequent shrubs were used in an attempt to spread the sampled shrubs throughout transects. If there were 2 shrubs equidistant from the previous shrub, the shrub closest to the center of the streambed or canyon was selected.

We selected 2 key browse species on the basis of palatability to ungulates and abundance in the study area: Salix spp. for Chaco Wash (wash use and wash control) and Atriplex canescens for side canyons (canyon use and canyon control). Percent use was assessed by examining 5 branches nearest to the observer's line of movement and between 0.25 and $2 \mathrm{~m}$ high. All current annual growth (previous years) on the chosen branch was examined for ungulate browsing. Only branches $0.25-2 \mathrm{~m}$ above ground were chosen because that height range is within $C$. elaphus and $O$. hemionus reach but too high for nonungulate herbivores. The number browsed out of the total number of shoots examined for each of the 5 branches was tallied for each shrub. We calculated mean browse levels for each of the 4 areas and compared browsing levels using analysis of variance [27]. We used stepwise multiple regression [27] to determine proportional use of browse species by C. elaphus and O. hemionus, with numbers of pellet 
groups/transect of each species used as predictor variables (see below) and browse levels as the outcome variable.

We assessed relative C. elaphus and O. hemionus use by counting all pellet groups encountered within $2 \mathrm{~m}$ of a straight line between shrubs used in browse surveys for the first $100 \mathrm{~m}$ of the browse transect. We identified pellet groups to species and totaled groups by species for each transect. We compared differences between areas using analysis of variance [27].

2.3. Grazing and Soil Surveys. We conducted grazing surveys in late August and early September, 2004 and 2006, at approximately the peak of graze species growth for the respective years. We compared 2 use and 1 control areas. The 2 use areas were the top of Chacra Mesa (CM), a Pinus edulisJuniperus spp. grassland, and the side ridges ("fingers") of mesa descending from the mesa top on the northeast side of CM (hereafter, Chacra Fingers (CF)). The control area included both South and West Mesas (SWM), which were Juniperus grasslands. We established $24100 \mathrm{~m}$ transects on CM and CF, respectively, 18 transects on South Mesa, and 6 on West Mesa, for a total of 72 transects. We randomly chose transect starting locations on a GIS basemap of Chaco. All 24 transects proceeded in the same direction in each area: toward $300^{\circ}$ on $\mathrm{CM}$ and $210^{\circ}$ on $\mathrm{CF}$, directions that roughly paralleled the run of the landscape, and $300^{\circ}$ on SWM to correspond with CM.

We tallied ground cover (i.e., soil, rock, plant, and cryptogamic crust) every $1 \mathrm{~m}$ along the $100 \mathrm{~m}$ transects. We identified all plants to species when possible and measured average leaf height (residual stubble height ( $\mathrm{RSH})$ ) of grasses by pulling the blades of grass up and measuring their height, not by measuring in situ [25]. We summarized RSHs obtained by species and compared these to published standards to determine degree of use (i.e., if any grass species in any of the areas were overgrazed [25]). If the cover was not grass, we located the nearest grass plant to the point and recorded its species and RSH. We calculated 95\% CIs around mean RSH to see if mean RSH differed from standards (e.g., if the standard was included in 95\% CIs, there was no difference).

Several soil types were present on each area, and transects occurred on a variety of soil types within each area. Consequently, we compared ground cover and type of plant cover (grass, forb, shrub, tree, and other) among sites using multivariate analysis of covariance [28], with mean herbaceous productivity of soils during average precipitation years (hereafter, productivity) as a covariate to account for differences in soils among transects and sites. For 2006 only, we used stepwise multiple regression [27] to model the proportional effects of C. elaphus and O. hemionus use on RSH of Bouteloua spp. on the CM, CF, and SWM areas. We determined relative use by $C$. elaphus and $O$. hemionus by tallying numbers of fecal pellet groups within $1 \mathrm{~m}$ of either side of the $100 \mathrm{~m}$ line transect (i.e., a $2 \times 100 \mathrm{~m}$ belt transect). We selected Bouteloua spp. as the test species because they represented $>51 \%$ of total grass cover on transects and were highly palatable to C. elaphus [29, 30]; the next most common species (Pleuraphis jamesii) comprised $<21 \%$. We included productivity in all regression models.
TABLE 1: Mean numbers of C. elaphus and O. hemionus pellet groups in use and control areas of Chaco Wash (Wash) and side canyons (Canyon), Chaco Culture National Historical Park, April 2004-2007.

\begin{tabular}{|c|c|c|c|c|c|c|}
\hline \multirow{2}{*}{ Year } & \multirow{2}{*}{ Treatment } & \multirow{2}{*}{ Canyon } & \multicolumn{2}{|c|}{ C. elaphus } & \multicolumn{2}{|c|}{ O. hemionus } \\
\hline & & & Groups & SE & Groups & SE \\
\hline \multirow{4}{*}{2004} & \multirow{2}{*}{ Control } & Wash & $0.1^{\mathrm{A}}$ & 0.3 & $0.2^{\mathrm{Y}}$ & 0.4 \\
\hline & & Canyon & $0^{\mathrm{A}}$ & 0 & $0.6^{\mathrm{Y}}$ & 0.7 \\
\hline & \multirow{2}{*}{ Use } & Wash & $7.9^{\mathrm{B}}$ & 2.6 & $1.5^{\mathrm{X}}$ & 1.3 \\
\hline & & Canyon & $6.0^{\mathrm{B}}$ & 3.7 & $2.5^{\mathrm{X}}$ & 2.6 \\
\hline \multirow{4}{*}{2005} & \multirow{2}{*}{ Control } & Wash & $0.3^{\mathrm{A}}$ & 0.2 & $1.9^{\mathrm{X}}$ & 0.5 \\
\hline & & Canyon & $2.3^{\mathrm{A}}$ & 0.9 & $8.9^{\mathrm{Y}}$ & 0.8 \\
\hline & \multirow{2}{*}{ Use } & Wash & $6.9^{\mathrm{B}}$ & 1.4 & $2.7^{\mathrm{X}}$ & 0.5 \\
\hline & & Canyon & $13.7^{\mathrm{C}}$ & 2.1 & $8.5^{\mathrm{Y}}$ & 0.8 \\
\hline \multirow{4}{*}{2006} & \multirow{2}{*}{ Control } & Wash & $0.6^{\mathrm{B}}$ & 0.3 & 0.2 & 0.3 \\
\hline & & Canyon & $0.3^{\mathrm{B}}$ & 0.2 & 1.2 & 0.8 \\
\hline & \multirow{2}{*}{ Use } & Wash & $3.6^{\mathrm{A}}$ & 1.1 & 0.4 & 0.4 \\
\hline & & Canyon & $4.2^{\mathrm{A}}$ & 1.0 & 2.4 & 1.2 \\
\hline \multirow{4}{*}{2007} & \multirow{2}{*}{ Control } & Wash & $0.6^{\mathrm{B}}$ & 0.3 & $4.0^{\mathrm{XY}}$ & 0.9 \\
\hline & & Canyon & $0.1^{\mathrm{B}}$ & 0.1 & $1.1^{\mathrm{Z}}$ & 0.3 \\
\hline & \multirow{2}{*}{ Use } & Wash & $2.9^{\mathrm{A}}$ & 0.9 & $5.1^{\mathrm{X}}$ & 1.0 \\
\hline & & Canyon & $3.2^{\mathrm{A}}$ & 0.5 & $2.4^{\mathrm{YZ}}$ & 0.3 \\
\hline
\end{tabular}
$0.05)$.

To assess invasion of grasslands by noxious weeds, we used cover of Gutierrezia sarothrae, an unpalatable subshrub indicative of overgrazing, and Bromus tectorum, an invasive exotic annual grass that increases with overgrazing [29]. We compared percent cover of these species among sites and between years (2004 and 2006) using Kruskal-Wallis tests [27]. Last, to assess possible herbivore-related trampling effects on soils, we measured water infiltration every $10 \mathrm{~m}$ along the $100 \mathrm{~m}$ transects. We determined relative infiltration rates by pouring $10 \mathrm{~mL}$ water into a clear graduated cylinder and timing how long it took for $2 \mathrm{~mL}$ of water to soak into the ground [31]. Timing was stopped at 30 seconds, and this test was not performed if the ground cover at that point was rock. We compared infiltration among sites using analysis of variance [27].

\section{Results}

3.1. Browsing Impacts. Numbers of $C$. elaphus pellet groups differed between use and control areas in all years $\left(F_{3, \geq 36} \geq\right.$ 7.9; $P \leq 0.001$ ) and were approximately 138X, 8X, 9X, and 9X greater in use areas, 2004-2007, respectively (Table 1). Control areas never differed in annual comparisons $(P \geq$ $0.298)$. Use areas only differed $(P=0.001)$ in 2005 when the canyon use area had approximately twice the relative use of the wash use area (Table 1$)$; no other years differed $(P \geq$ 0.347).

Numbers of $O$. hemionus pellet groups differed $\left(F_{3, \geq 40} \geq\right.$ 5.0; $P \leq 0.002)$ among use and control areas in all years except $2006\left(F_{3,40}=2.0 ; P=0.127\right)$. In contrast to C. elaphus, relative use by $O$. hemionus was variable and showed no 
Table 2: Percent utilization (SE) of Salix spp. in Chaco Wash and Atriplex canescens in the side canyons in use and control areas, Chaco Culture National Historical Park, April 2004-2007.

\begin{tabular}{lcccccccc}
\hline & \multicolumn{4}{c}{ Salix spp. } & \multicolumn{3}{c}{ Atriplex canescens } \\
Year & \multicolumn{2}{c}{ Use } & \multicolumn{2}{c}{ Control } & \multicolumn{2}{c}{ Use } & \multicolumn{2}{c}{ Control } \\
& $\%$ & SE & $\%$ & SE & $\%$ & SE & $\%$ & SE \\
\hline 2004 & $8.8^{\mathrm{A}}$ & 2.1 & $0^{\mathrm{B}}$ & 0 & $25.3^{\mathrm{X}}$ & 2.5 & $0^{\mathrm{Y}}$ & 0 \\
2005 & 5.8 & 2.0 & 7.4 & 0.7 & $20.6^{\mathrm{X}}$ & 2.9 & $14.4^{\mathrm{Y}}$ & 2.4 \\
2006 & 12.6 & 3.3 & 8.7 & 0.8 & $31.7^{\mathrm{X}}$ & 2.6 & $24.4^{\mathrm{Y}}$ & 2.3 \\
2007 & $14.9^{\mathrm{A}}$ & 2.4 & $8.5^{\mathrm{B}}$ & 1.5 & $12.4^{\mathrm{X}}$ & 2.0 & $3.0^{\mathrm{Y}}$ & 1.0 \\
\hline AB and XY Different letters & within a species and year indicate significant \\
differences $(P<0.05)$. & \multicolumn{4}{l}{$l$}
\end{tabular}

consistent patterns among years (Table 1); in 2004 use areas showed approximately $5 \mathrm{X}$ greater relative use than controls; in 2005 , side canyons showed approximately $4 \mathrm{X}$ greater use than Chaco Wash; but in 2007 there was a tendency for Chaco Wash to show approximately $3 \mathrm{X}$ greater use than the side canyons (Table 1).

Salix spp. use was significantly higher $\left(F_{1,18} \geq 5.1 ; P \leq\right.$ $0.037)$ in the wash use area than in the wash control in 2004 and 2007, but not in 2005 or $2006\left(F_{1,18} \leq 2.1 ; P \geq 0.162\right)$ (Table 2). Use of Atriplex canescens was significantly higher $\left(F_{1,18} \geq 4.5 ; P \leq 0.049\right)$ in the canyon use areas than in the canyon controls in all 4 years (Table 2 ).

Both C. elaphus $(r=0.372-0.402 ; P=0.006-0.031)$ and $O$. hemionus $(r=0.457-0.795 ; P \leq 0.001-0.013)$ use was correlated to browse utilization, 2004-2007. In 20042007, models (2004: $F_{1,27}=7.1 ; P=0.013 ; 2005: F_{1,37}=63.4$; $P<0.0001 ; 2006: F_{1,36}=42.1 ; P<0.0001 ; 2007: F_{1,38}=$ 37.1; $P<0.0001$ ) of browse use included only $O$. hemionus use and accounted for $20.8 \%, 63.1 \%, 53.9 \%$, and $49.4 \%$ of the variation in browse utilization, 2004-2007, respectively. Forcing C. elaphus use into the model resulted in only an additional $1.3 \%, 0.6 \%, 2.7 \%$, and $0.8 \%$ of the variation in browsing levels being accounted for, 2004-2007, respectively.

3.2. Grazing Impacts. CM, CF, and SWM differed $\left(F_{4,66}=\right.$ 20.1; $P<0.001)$ in ground cover, including live plants $\left(F_{2,68}=\right.$ 16.1; $P<0.001)$, bare soil $\left(F_{2,68}=11.2 ; P<0.001\right)$, rock $\left(F_{2,68}=21.2 ; P<0.001\right)$, and cryptogamic crusts $\left(F_{2,68}=9.5\right.$; $P<0.001$ ) in 2004 (Table 3 ). Ground cover similarly varied in $2006\left(F_{4,64}=3.5 ; P=0.013\right)$ for live plants $\left(F_{2,67}=3.3\right.$; $P=0.043)$, bare soil $\left(F_{2,67}=5.0 ; P=0.010\right)$, and $\operatorname{rock}\left(F_{2,67}=\right.$ 9.4; $P<0.001)$, but not cryptogamic crusts $\left(F_{2,67}=0.4\right.$; $P=0.702)$. In both years, CM $(P \leq 0.009)$ had significantly more plant cover than CF or SWM, which were similar each year $(P \geq 0.459)$ (Table 3$)$.

$\mathrm{CM}, \mathrm{CF}$, and SWM similarly varied in live plant cover (2004: $\left.F_{5,65}=9.8 ; P<0.001 ; 2006: F_{5,61}=3.3 ; P=0.003\right)$. Sites consistently differed in cover of grasses $\left(2004: F_{2,68}=\right.$ 18.7; $\left.P<0.001 ; 2006: F_{2,64}=4.8 ; P=0.011\right)$, forbs $(2004$ : $\left.F_{2,68}=6.8 ; P=0.002 ; 2006: F_{2,64}=2.5 ; P=0.094\right)$, and trees (2004: $\left.F_{2,68}=8.2 ; P=0.001 ; 2006: F_{2,64}=12.2 ; P<0.001\right)$, but not shrubs (2004: $F_{2,68}=5.7 ; P=0.005 ; 2006: F_{2,64}=1.1$; $P=0.339$ ) or other vegetation (primarily succulents; 2004 : $\left.F_{2,68}=0.9 ; P=0.398 ; 2006: F_{2,64}=1.2 ; P=0.311\right) . \mathrm{CM}$
TABLE 3: Mean percent cover of plants, cryptogamic crust, bare soil, and rock in use (Chacra Mesa, Chacra Fingers) and control (South/West Mesas) areas, Chaco Culture National Historical Park, August-September 2004 and 2006.

\begin{tabular}{|c|c|c|c|c|c|c|c|c|}
\hline \multirow{2}{*}{ Area } & \multicolumn{2}{|c|}{ Plant } & \multicolumn{2}{|c|}{ Crust } & \multicolumn{2}{|c|}{ Bare soil } & \multicolumn{2}{|c|}{ Rock } \\
\hline & $\%$ & SE & $\%$ & SE & $\%$ & SE & $\%$ & SE \\
\hline \multicolumn{9}{|l|}{2004} \\
\hline Chacra Mesa & $42.9^{\mathrm{A}}$ & 1.6 & $0.0^{\mathrm{B}}$ & 0.0 & $57.1^{\mathrm{B}}$ & 1.6 & $0.0^{\mathrm{C}}$ & 0.0 \\
\hline Chacra Fingers & $19.4^{\mathrm{B}}$ & 1.7 & $4.0^{\mathrm{A}}$ & 1.1 & $52.5^{\mathrm{B}}$ & 3.9 & $24.1^{\mathrm{A}}$ & 4.7 \\
\hline $\begin{array}{l}\text { South/West } \\
\text { Mesas }\end{array}$ & $20.0^{\mathrm{B}}$ & 1.9 & $0.7^{\mathrm{B}}$ & 0.2 & $71.6^{\mathrm{A}}$ & 2.1 & $7.7^{\mathrm{B}}$ & 2.1 \\
\hline \multicolumn{9}{|l|}{2006} \\
\hline Chacra Mesa & $28.4^{\mathrm{A}}$ & 2.1 & 0.00 & 0.00 & $66.2^{\mathrm{A}}$ & 2.7 & $3.5^{\mathrm{A}}$ & 2.7 \\
\hline Chacra Fingers & $17.2^{\mathrm{B}}$ & 2.4 & 0.13 & 0.07 & $53.5^{\mathrm{B}}$ & 4.0 & $29.3^{\mathrm{B}}$ & 5.2 \\
\hline $\begin{array}{l}\text { South/West } \\
\text { Mesas }\end{array}$ & $19.6^{\mathrm{B}}$ & 2.5 & 0.61 & 0.33 & $59.0^{\mathrm{AB}}$ & 4.2 & $19.6^{\mathrm{B}}$ & 4.4 \\
\hline
\end{tabular}

always had $>2 \mathrm{X}$ the grass cover $(P \leq 0.028)$ of the other sites, and forb cover was also greater or similar to the other areas. Conversely, tree cover was less or similar on CM (Table 4). Residual stubble heights of all grass species in each area each year were generally much higher than recommended use standards (Table 5), and no grass species was overutilized in any year based upon published standards [25].

Relative use by $C$. elaphus was greater $\left(F_{2,67}=15.1\right.$; $P<0.001)$ on CM (5.5 (SE = 0.9) groups/transect) and CF $(5.8(\mathrm{SE}=1.0)$ groups/transect) than SWM $(0.4(\mathrm{SE}=$ 0.1 ) groups/transect). Conversely, relative use by $O$. hemionus was greater $\left(F_{2,67}=5.1 ; P=0.009\right)$ on $\mathrm{CF}(4.1(\mathrm{SE}=0.7)$ groups/transect) and SWM (3.2 (SE =0.8) groups/transect) than CM (1.7 $(\mathrm{SE}=0.4)$ groups/transect). Residual stubble height of Bouteloua spp. was not related to either C. elaphus $\left(F_{1,64}=0.7 ; P=0.411\right)$ or $O$. hemionus $\left(F_{1,64}=0.1 ; P=\right.$ $0.718)$ relative use but was related to site productivity $\left(F_{1,64}=\right.$ 26.6; $P<0.001)$ and the $C$. elaphus $\times$ site interaction $\left(F_{2,64}=7.0 ; P=0.002\right)$. Consequently, we looked at the 3 sites independently to assess relative C. elaphus use. On $\mathrm{CM}$, relative $C$. elaphus use was positively related to RSH of Bouteloua spp. $\left(F_{1,21}=10.5 ; P=0.005 ; \beta=0.160(\mathrm{SE}=0.054)\right)$, whereas relative use by $C$. elaphus and RSH of Bouteloua spp. were not related on $\operatorname{CF}\left(F_{1,20}=1.3 ; P=0.267\right)$ or $\operatorname{SWM}\left(F_{1,18}=\right.$ $0.02 ; P=0.879)$. Relative use by $C$. elaphus accounted for $33 \%$ of the variation in RSH on CM. Relative use by $O$. hemionus was unrelated to RSH of Bouteloua spp. on all sites $(P \leq$ $0.414)$.

In 2004, there was more Gutierrezia sarothrae $\left(H_{2}=24.5\right.$; $P<0.001)$ on CM $(2.0 \%(\mathrm{SE}=0.3)$ of total plant cover; $P \leq$ $0.001)$ than on the CF $(0.3 \%(\mathrm{SE}=0.1))$ or SWM $(0.5 \%(\mathrm{SD}=$ $0.3)$, which did not differ $(P=0.551)$. Bromus tectorum did not vary $\left(H_{2}=0.7 ; P=0.690\right)$ in total cover among $\mathrm{CF}(0.29 \%$ $(\mathrm{SE}=0.16)), \mathrm{CM}(0.00 \%(\mathrm{SE}=0.00))$, or SWM $(0.17 \%(\mathrm{SE}=$ $0.10))$. Similarly, Gutierrezia sarothrae varied $\left(H_{2}=16.0 ; P=\right.$ $0.0003)$ among sites in 2006 and was more common $(2.5 \%$ $(\mathrm{SE}=0.5) ; P \leq 0.002)$ on $\mathrm{CM}$ than on $\mathrm{CF}(0.8 \%(\mathrm{SE}=0.3))$ or 
TABLE 4: Mean percent of total plant cover comprised of grasses, forbs, shrubs, trees, and other (i.e., succulents, etc.) classes of plants in use (Chacra Mesa, Chacra Fingers) and control (South/West Mesas) areas, Chaco Culture National Historical Park, August-September 2004 and 2006.

\begin{tabular}{|c|c|c|c|c|c|c|c|c|c|c|}
\hline \multirow{2}{*}{ Area } & \multicolumn{2}{|c|}{ Grass } & \multicolumn{2}{|c|}{ Forb } & \multicolumn{2}{|c|}{ Shrub } & \multicolumn{2}{|c|}{ Tree } & \multicolumn{2}{|c|}{ Other } \\
\hline & $\%$ & SE & $\%$ & SE & $\%$ & SE & $\%$ & SE & $\%$ & SE \\
\hline \multicolumn{11}{|l|}{2004} \\
\hline Chacra Mesa & $22.5^{\mathrm{A}}$ & 1.0 & $6.2^{\mathrm{A}}$ & 1.1 & $13.0^{\mathrm{A}}$ & 1.0 & $0.9^{\mathrm{B}}$ & 0.8 & 0.3 & 0.1 \\
\hline Chacra Fingers & $6.1^{\mathrm{C}}$ & 0.8 & $0.9^{\mathrm{B}}$ & 0.3 & $7.2^{\mathrm{B}}$ & 0.8 & $4.6^{\mathrm{A}}$ & 0.9 & 0.6 & 0.2 \\
\hline South/West Mesas & $8.4^{\mathrm{B}}$ & 0.7 & $2.5^{\mathrm{A}}$ & 0.6 & $7.8^{\mathrm{B}}$ & 0.8 & $1.0^{\mathrm{B}}$ & 0.7 & 0.4 & 0.2 \\
\hline \multicolumn{11}{|l|}{2006} \\
\hline Chacra Mesa & $21.0^{\mathrm{A}}$ & 1.9 & $1.3^{\mathrm{AB}}$ & 0.3 & 5.9 & 0.7 & $0.1^{\mathrm{B}}$ & 0.3 & 0.04 & 0.04 \\
\hline Chacra Fingers & $8.5^{\mathrm{B}}$ & 1.7 & $0.9^{\mathrm{B}}$ & 0.5 & 6.2 & 0.6 & $1.5^{\mathrm{A}}$ & 0.2 & 0.04 & 0.04 \\
\hline South/West Mesas & $8.7^{\mathrm{B}}$ & 2.0 & $1.7^{\mathrm{A}}$ & 0.6 & 4.8 & 0.9 & $0.2^{\mathrm{B}}$ & 0.1 & 0.13 & 0.08 \\
\hline
\end{tabular}

${ }^{\mathrm{ABC}}$ Different letters within a column and year indicate significant differences $(P<0.05)$.

TABLE 5: Species, number of plants per species, and mean residual leaf stubble height $(\mathrm{cm})$ with $95 \%$ confidence interval for grasses detected on grazing surveys, Chaco Culture National Historical Park, 2004 and 2006. The cutoff height below which a plant is considered "overgrazed," as defined by Allison et al. (2007) [25], is given (Standard).

\begin{tabular}{|c|c|c|c|c|c|c|c|c|}
\hline \multirow{2}{*}{ Area } & \multirow{2}{*}{ Species } & \multicolumn{3}{|c|}{2004} & \multicolumn{3}{|c|}{2006} & \multirow{2}{*}{ Standard } \\
\hline & & $\mathrm{RSH}$ & $95 \% \mathrm{CI}$ & $N$ & $\mathrm{RSH}$ & $95 \% \mathrm{CI}$ & $N$ & \\
\hline Chacra Fingers & Sporobolus airoides & 12.5 & $11.4-13.6$ & 168 & 24.4 & $22.1-26.7$ & 38 & $\mathrm{n} / \mathrm{a}$ \\
\hline Chacra Fingers & Bouteloua spp. & 8.1 & $7.7-8.5$ & 643 & 6.3 & $6.1-6.6$ & 302 & 6.35 \\
\hline Chacra Fingers & Bouteloua eriopoda & - & - & - & 6.4 & $5.3-7.5$ & 35 & 6.35 \\
\hline Chacra Fingers & Bouteloua gracilis & - & - & - & 7.7 & $5.9-8.4$ & 25 & 3.81 \\
\hline Chacra Fingers & Pleuraphis jamesii & 13.2 & $12.2-14.1$ & 211 & 13.8 & $12.9-14.8$ & 107 & 6.35 \\
\hline Chacra Fingers & Muhlenbergia spp. & 23.3 & $19.4-27.1$ & 11 & - & - & - & 6.35 \\
\hline Chacra Fingers & Achnatherum hymenoides & 22.2 & $21.4-23.1$ & 542 & 21.2 & $19.6-22.8$ & 44 & 10.16 \\
\hline Chacra Fingers & Muhlenbergia torreyi & 7.0 & $4.3-9.7$ & 13 & 10.6 & $7.7-13.4$ & 18 & 1.91 \\
\hline Chacra Fingers & Sporobolus cryptandrus & 21.9 & $16.6-27.2$ & 22 & 15.8 & $12.7-18.8$ & 13 & 10.16 \\
\hline Chacra Fingers & Hesperostipa comata & 23.8 & $22.7-25.0$ & 250 & 18.8 & $16.6-21.2$ & 28 & 10.16 \\
\hline Chacra Fingers & Aristida spp. & 12.4 & $10.9-13.8$ & 80 & - & - & - & 6.35 \\
\hline Chacra Mesa & Bouteloua gracilis & 6.9 & $6.2-7.6$ & 127 & - & - & - & 3.81 \\
\hline Chacra Mesa & Bouteloua spp. & 7.2 & $6.8-7.7$ & 522 & 7.2 & $7.0-7.4$ & 456 & 6.35 \\
\hline Chacra Mesa & Achnatherum hymenoides & 21.2 & $20.6-21.9$ & 1032 & 19.5 & $18.9-20.1$ & 173 & 10.16 \\
\hline Chacra Mesa & Muhlenbergia torreyi & 8.5 & $5.1-12.0$ & 13 & 8.7 & $6.9-10.5$ & 32 & 1.91 \\
\hline Chacra Mesa & Sporobolus cryptandrus & 15.9 & $13.0-18.7$ & 28 & 11.8 & $11.1-12.8$ & 117 & 10.16 \\
\hline Chacra Mesa & Elymus elymoides & 8.6 & $7.3-9.8$ & 11 & - & - & - & 10.16 \\
\hline Chacra Mesa & Hesperostipa comata & 30.0 & $29.0-30.9$ & 603 & 15.9 & $11.7-20.0$ & 80 & 10.16 \\
\hline Chacra Mesa & Pleuraphis jamesii & - & - & - & 13.9 & $13.1-14.7$ & 61 & 6.35 \\
\hline S/W Mesas & Sporobolus airoides & 2243 & $19.1-25.6$ & 38 & 29.5 & $26.8-32.2$ & 21 & $\mathrm{n} / \mathrm{a}$ \\
\hline S/W Mesas & Bouteloua spp. & 8.4 & $7.9-8.9$ & 253 & 13.5 & $12.7-14.2$ & 246 & 6.35 \\
\hline S/W Mesas & Bouteloua gracilis & - & - & - & 13.0 & $11.2-14.7$ & 71 & 3.81 \\
\hline S/W Mesas & Bouteloua eriopoda & - & - & - & 13.7 & $12.2-15.2$ & 59 & 6.35 \\
\hline S/W Mesas & Bouteloua curtipendula & - & - & - & 16.0 & $14.9-17.1$ & 48 & 10.16 \\
\hline S/W Mesas & Pleuraphis jamesii & 15.4 & $14.7-16.0$ & 302 & 20.1 & $19.4-20.8$ & 381 & 6.35 \\
\hline S/W Mesas & Muhlenbergia spp. & 16.2 & $12.1-20.2$ & 17 & 19.3 & $15.3-23.3$ & 13 & 6.35 \\
\hline S/W Mesas & Achnatherum hymenoides & 28.9 & $27.9-29.9$ & 281 & 28.3 & $27.1-29.6$ & 231 & 10.16 \\
\hline S/W Mesas & Hesperostipa comata & 31.4 & $29.3-33.0$ & 318 & 24.0 & $22.3-25.6$ & 123 & 10.16 \\
\hline S/W Mesas & Aristida spp. & 12.9 & $11.6-14.2$ & 19 & - & - & - & 6.35 \\
\hline S/W Mesas & Sporobolus cryptandrus & - & - & - & 24.9 & $22.2-27.6$ & 30 & 10.16 \\
\hline
\end{tabular}


TABLE 6: Mean water infiltration time (sec) in 3 graze survey areas, Chaco Culture National Historic Park, 2004 and 2006.

\begin{tabular}{lcccc}
\hline \multirow{2}{*}{ Area } & \multicolumn{2}{c}{2004} & \multicolumn{2}{c}{2006} \\
& Time & SE & Time & SE \\
\hline Chacra Mesa & $7.68^{\mathrm{A}}$ & 0.4 & 11.0 & 0.8 \\
Chacra Fingers & $9.93^{\mathrm{B}}$ & 0.6 & 9.9 & 1.1 \\
South/West Mesas & $9.31^{\mathrm{B}}$ & 0.6 & 10.6 & 0.9 \\
\hline $\mathrm{AB}$ Times followed by the same letter do not differ $(P>0.05)$. &
\end{tabular}

${ }^{\mathrm{AB}}$ Times followed by the same letter do not differ $(P>0.05)$.

on SWM $(0.3 \%(\mathrm{SE}=0.2))$, which did not differ $(P=0.326)$. Cover of Bromus tectorum did not vary $\left(\mathrm{H}_{2}=2.8 ; P=0.245\right)$ among $\mathrm{CM}(0.0 \%$; $\mathrm{SE}=0.0), \mathrm{CF}(0.16 \% ; \mathrm{SE}=0.12)$, and $\mathrm{SWM}$ $(0.37 \%$; SE $=0.14)$ in 2006.

Last, cover of Gutierrezia sarothrae increased on CM from 2004 to $2006\left(H_{1}=-4.6 ; P<0.001\right)$, but cover remained similar on CF and SWM $\left(H_{1} \leq-0.2 ; P \geq 0.854\right)$. Cover of Bromus tectorum did not vary within areas between years $(H \leq-0.8 ; P \geq 0.406)$.

3.3. Infiltration Rates. Water infiltration rates differed $\left(F_{2,69}\right.$ $=4.31 ; P=0.017$ ) among sites in 2004 (Table 6). Infiltration rate on CM was faster than CF $(P=0.006)$ and SWM $(P=$ $0.043)$, which did not differ $(P=0.438$; Table 6). In 2006, water infiltration times $\left(F_{2,67}=2.2 ; P=0.122\right)$ were similar among sites (Table 6).

\section{Discussion}

C. elaphus use had little apparent impact on plants or soils of Chaco, and levels of browsing (Table 2) and grazing (as indicated by high RSHs; Table 5) were low. Rather than negatively impacting plant communities, some evidence suggests that the low use of CM may have resulted in an C. elaphus-induced optimization [11, 12] of CM grasslands, as residual biomass of the most abundant palatable graminoid, Bouteloua spp., increased with increasing $C$. elaphus grazing even after accounting for soil productivity. Lack of impacts and possible facilitation of CM grasslands by C. elaphus was likely a consequence of low C. elaphus densities $\left(0.2-0.4 / \mathrm{km}^{2}\right)$ during this project. Previously, C. elaphus optimization of forested grasslands in the Blue Mountains of Oregon was seen at densities of generally $<15 / \mathrm{km}^{2}[12]$.

C. elaphus use averaged $>7 \mathrm{X}$ greater in the wash and canyon use areas compared to controls (Table 1), whereas use by $O$. hemionus was more variable and not directly tied to use and control areas designated from C. elaphus distribution (Table 1). Use of Salix spp. was variable but generally greater in use areas than controls, although this difference was significant only in 2004 and 2007 (Table 2). However, maximum use of Salix spp. in Chaco was $\leq 15 \%$ annually, and use of Atriplex canescens was $<32 \%$ annually (Table 2). Even the highest utilization rate observed was far below levels affecting plant viability; for example, riparian shrubs can sustain use of 50-60\% without impacts on plant health and structure [32]. In areas where C. elaphus have been implicated as having strong impacts on plant communities, browse utilization was much greater than on Chaco. For example, Salix spp. use in Rocky Mountain National Park ranged from approximately 70 to 90\% [10] and Populus tremuloides use averaged from 77 to $90 \%$ in Yellowstone National Park [20]. Moreover, O. hemionus, not C. elaphus, were responsible for the majority of browsing correlated with ungulate use in Chaco (21-63\% of variance).

Similar to browse use, effects of C. elaphus grazing on grass species were undetectable on Chaco. Based on RSHs, no differences in grazing intensity were apparent among the 3 areas surveyed, and overall grazing pressure was light in all areas (Table 5). Moreover, overall plant cover was greatest on CM, a use area; bare soil was highest (2004) or similar (2006) on SWM, a control area; cryptogamic crust cover was greatest (2004) or similar (2006) on CF, a use area (Table 3); and water infiltration in 2004 was fastest on CM, a use area (infiltration rate did not differ among areas in 2006); each of these indicates little or no effect of C. elaphus grazing or trampling on plant cover, cryptogamic crusts, or soil compaction [30, $33,34,36]$. Of the 3 areas, CF and CM received the highest relative $C$. elaphus use (5.8 and 5.5 pellet groups/transect, resp., versus 0.4 on SWM) and CF also received the highest use by $O$. hemionus (4.1 pellet groups/transect versus 3.2 on SWM and 1.7 on CM); thus, CF would be expected to show the largest effect on infiltration rates if C. elaphus or combined ungulate trampling was compacting soils on Chaco. Further, the CF area had the highest coverage of cryptogamic crusts in 2004 (Table 3), and cryptogamic crusts would be expected to be negatively impacted by $C$. elaphus trampling.

Similarly, there were no trends in noxious species that were attributable to patterns of C. elaphus use. Although Gutierrezia sarothrae increased from 2004 (2.0\% cover) to 2006 (2.5\% cover) on CM, if this increase was attributable to disturbance associated with C. elaphus trampling $[33,34]$ similar or greater increases should have been seen on CF, as C. elaphus utilized CF similarly to CM (5.8 versus 5.5 pellet groups/transect) and $O$. hemionus used CF more (4.1 versus 1.7 pellet groups/transect, resp.), but no differences were detected. Because annual changes in plant demographics can be large in arid systems [33-35] independent of herbivore effects, the observed change in cover of Gutierrezia sarothrae likely was related to annual weather or other factors as it was not associated with any trends in C. elaphus use.

In contrast to negative impacts, there was some evidence that the light grazing seen in Chaco stimulated grassland production. On CM, RSH of Bouteloua spp. was positively associated with degree of $C$. elaphus use, indicating that RSHs increased as C. elaphus grazing increased; that is, Bouteloua spp. may have shown compensatory increases in aboveground biomass due to C. elaphus herbivory. Herbivore optimization of herbaceous plants by light C. elaphus grazing has been demonstrated experimentally [12]. Herbivore optimization occurs because of compensatory growth of plants due to light removal of existing tissue, perhaps augmented by fertilization from urine and feces and removal of coarse, less photosynthetically active tissue and lessening of competition $[11,12,36]$. Through these processes, light to moderate grazing can increase net annual primary production of grasslands [11, 36]. On CM, residual biomass of Bouteloua spp., 
as measured by RSHs, was positively related to the degree of C. elaphus use. Further, cover of grasses and forbs was greater in areas receiving more C. elaphus use (Table 4), and plant diversity was highest in the heaviest ungulate use area of Chaco (CF). Collectively, these results further indicate that herbivory by $C$. elaphus (or combined herbivory by $C$. elaphus and O. hemionus) was not negatively impacting the desert grasslands of Chaco and suggest that herbivory may have been enhancing the diversity and productivity of these grasslands.

In conclusion, the presence of C. elaphus alone does not mean that deleterious impacts on plant communities will be present, even in protected areas. C. elaphus occurred in and around Chaco in low densities $\left(0.2-0.4 / \mathrm{km}^{2}\right)$ and showed minimal or no impacts on plants or soils and may have stimulated plant growth by light grazing $[11,36]$. Additionally, viewing of $C$. elaphus was a highly valued experience for visitors of Chaco and such opportunities would be lost if $C$. elaphus were culled because of anticipated negative impacts on plant communities such as seen in other western national parks in the USA. Thus, at current population levels, no actions to decrease C. elaphus numbers in the Chaco area are warranted if driven by concerns over effects of C. elaphus herbivory.

\section{Conflict of Interests}

The authors declare that there is no conflict of interests regarding the publication of this paper.

\section{Acknowledgments}

The authors thank the U.S. National Park Service and the New Mexico State University, Agricultural Experiment Station for funding this project. T. Kamienski, B. Keller, B. Krueger, L. Lomas, B. Shattuck, and E. Watters provided valuable field and logistical assistance. All activities in this project were in accordance with NMSU Institutional Animal Use and Care Permit 2002-027.

\section{References}

[1] S. W. Chadde and C. E. Kay, "Tall-willow communities on Yellowstone's Northern range: a test of the 'natural-regulation' paradigm," in The Greater Yellowstone Ecosystem: Redefining America's Wilderness Heritage, R. B. Keiter and M. S. Boyce, Eds., pp. 231-261, Yale University Press, New Haven, Conn, USA, 1991.

[2] F. J. Singer, L. C. Mark, and R. C. Cates, "Ungulate herbivory of willows on Yellowstone's northern winter range," Journal of Range Management, vol. 47, no. 6, pp. 435-443, 1994.

[3] F. J. Singer and R. A. Renkin, "Effects of browsing by native ungulates on the shrubs in big sagebrush communities in Yellowstone National Park," Great Basin Naturalist, vol. 55, no. 3, pp. 201-212, 1995.

[4] W. J. Ripple and E. J. Larsen, "Historic aspen recruitment, elk, and wolves in northern Yellowstone National Park, USA," Biological Conservation, vol. 95, no. 3, pp. 361-370, 2000.
[5] W. J. Ripple, E. J. Larsen, R. A. Renkin, and D. W. Smith, "Trophic cascades among wolves, elk, and aspen on Yellowstone National Park's northern range," Biological Conservation, vol. 102, pp. 227-234, 2001.

[6] H. R. Peinetti, M. A. Kalkhan, and M. B. Coughenour, "Longterm changes in willow spatial distribution on the elk winter range of Rocky Mountain National Park (USA)," Landscape Ecology, vol. 17, no. 4, pp. 341-354, 2002.

[7] M. Schütz, A. C. Risch, E. Leuzinger, B. O. Krüsi, and G. Achermann, "Impact of herbivory by red deer (Cervus elaphus L.) on patterns and processes in subalpine grasslands in the Swiss National Park," Forest Ecology and Management, vol. 181, no. 1-2, pp. 177-188, 2003.

[8] P. E. Packer, "Effects of trampling disturbance on watershed conditions, runoff, and erosion," Journal of Forestry, vol. 51, pp. 28-31, 1953.

[9] P. E. Packer, "Soil stability requirements for the gallatin elk winter range," The Journal of Wildlife Management, vol. 27, no. 3, p. 401, 1963.

[10] L. C. Zeigenfuss, F. J. Singer, and D. Bowden, "Vegetation responses to natural regulation of elk in Rocky Mountain National Park," in Ecological Evaluation of the Abundance and Effects of Elk Herbivory in Rocky Mountain National Park, Colorado, 1994-1999, F. J. Singer and L. C. Zeigenfuss, Eds., Open File Report 02-208, pp. 47-70, U. S. Geological Survey, Ft. Collins, Colo, USA, 2002.

[11] S. J. McNaughton, "Grazing as an optimization process: grassungulate relationships in the Serengeti," The American Naturalist, vol. 113, no. 5, pp. 691-703, 1979.

[12] K. M. Stewart, R. T. Bowyer, R. W. Ruess, B. L. Dick, and J. G. Kie, "Herbivore optimization by North American elk: consequences for theory and management," Wildlife Monographs, no. 167, pp. 1-24, 2006.

[13] N. J. Georgiadis, R. W. Ruess, S. J. McNaughton, and D. Western, "Ecological conditions that determine when grazing stimulates grass production," Oecologia, vol. 81, no. 3, pp. 316-322, 1989.

[14] E. W. Hamilton III, M. S. Giovannini, S. A. Moses, J. S. Coleman, and S. J. McNaughton, "Biomass and mineral element responses of a Serengeti short-grass species to nitrogen supply and defoliation: compensation requires a critical [N]," Oecologia, vol. 116, no. 3, pp. 407-418, 1998.

[15] C. De Mazancourt, M. Loreau, and L. Abbadie, "Grazing optimization and nutrient cycling: when do herbivores enhance plant production?" Ecology, vol. 79, no. 7, pp. 2242-2252, 1998.

[16] K. P. Alstad, J. M. Welker, S. A. Williams, and M. J. Trlica, "Carbon and water relations of Salix monticola in response to winter browsing and changes in surface water hydrology: an isotopic study using $\delta^{13} \mathrm{C}$ and $\delta^{18} \mathrm{O}$," Oecologia, vol. 120 , no. 3 , pp. 375-385, 1999.

[17] P. Török, O. Valkó, B. Deák, A. Kelemen, and B. Tóthmérész, "Traditional cattle grazing in a mosaic alkali landscape: effects on grassland biodiversity along a moisture gradient," PLOS ONE, vol. 9, no. 5, Article ID e97095, 2014.

[18] M. A. Relva and T. T. Veblen, "Impacts of introduced large herbivores on Austrocedrus chilensis forests in northern Patagonia, Argentina," Forest Ecology and Management, vol. 108, no. 1-2, pp. 27-40, 1998.

[19] C. E. Kay and D. L. Bartos, "Ungulate herbivory on Utah aspen: assessment of long-term exclosures," Journal of Range Management, vol. 53, no. 2, pp. 145-153, 2000. 
[20] National Research Council, Ecological dynamics on Yellowstone's Northern Range, National Academy Press, Washington, DC, USA, 2002.

[21] F. J. Singer and L. C. Zeigenfuss, "Ecological evaluation of the abundance and effects of elk herbivory in Rocky Mountain National Park, Colorado, 1994-1999," U.S. Geological Survey Open File Report 02-208, U.S. Geological Survey, Ft. Collins, Colo, USA, 2002.

[22] National Park Service, "Chaco culture national historical park: grazing history," Report, US National Park Service, Chaco Culture National Historic Park, Nageezi, NM, USA, 1998.

[23] M. L. Floyd, T. L. Fleischner, D. Hanna, and P. Whitefield, "Effects of historic livestock grazing on vegetation at Chaco Culture National Historic Park, New Mexico," Conservation Biology, vol. 17, no. 6, pp. 1703-1711, 2003.

[24] L. C. Bender and J. R. Piasecke, "Population demographics and dynamics of colonizing elk in a desert grassland-scrubland," Journal of Fish and Wildife Management, vol. 1, no. 2, pp. 152$160,2010$.

[25] C. D. Allison, J. L. Holechek, T. T. Baker, J. C. Boren, N. K. Ashcroft, and J. M. Fowler, "Rapid assessment methodology for proactive rangeland management," Rangelands, vol. 29, no. 2, pp. 45-50, 2007.

[26] Wyoming Game and Fish, Handbook of Biological Techniques, Wyoming Game and Fish Department, Laramie, Wyo, USA, 1982.

[27] J. H. Zar, Biostatistical Analysis, Prentice-Hall, Upper Saddle River, NJ, USA, 1996.

[28] D. F. Morrison, Multivariate Statistical Methods, McGraw-Hill, New York, NY, USA, 1990.

[29] J. Stubbendieck, S. L. Hatch, and C. H. Butterfield, North American Range Plants, University of Nebraska Press, Lincoln, Neb, USA, 4th edition, 1992.

[30] G. L. Wolters, "Elk effects on Bandelier National Monument meadows and grasslands," in Fire Effects in Southwestern Forests: Proceedings of the Second La Mesa Fire Symposium, C. D. Allen, Ed., US Forest Service General Technical Report RM-GTR-286, pp. 196-205, US Forest Service, Fort Collins, Colo, USA, 1996.

[31] H. Bouwer, "Intake rate: cylinder infiltrometer," in Methods of Soil Analysis Part 1: Physical and Mineralogical Methods, A. Klute, Ed., vol. 9 of Agronomy, pp. 825-843, American Society of Agronomy, Madison, Madison, Wis, USA, 1986.

[32] J. C. Moseley, P. S. Cook, A. J. Griffis, and J. O'Laughlin, "Guidelines for managing cattle grazing in riparian areas to protect water quality: review of research and best management practices policy," Report 15, Idaho Forest, Wildlife, and Range Policy Analysis Group, University of Idaho, Moscow, Idaho, USA, 1997.

[33] S. P. Rupp, M. C. Wallace, D. Wester, S. Fettig, and R. Mitchell, "Effects of simulated elk grazing and trampling (I): intensity," Alces, vol. 37, pp. 129-146, 2001.

[34] S. P. Rupp, M. C. Wallace, D. Wester, S. Fettig, and R. Mitchell, "Effects of simulated elk grazing and trampling (II): frequency," Alces, vol. 37, pp. 147-161, 2001.

[35] P. R. Kemp, "Phenological patterns of Chihuahuan desert plants in relation to the timing of water availability," Journal of Ecology, vol. 71, no. 2, pp. 427-436, 1983.

[36] M. Vavra, "Livestock grazing and wildlife: developing compatibilities," Rangeland Ecology and Management, vol. 58, no. 2, pp. 128-134, 2005. 

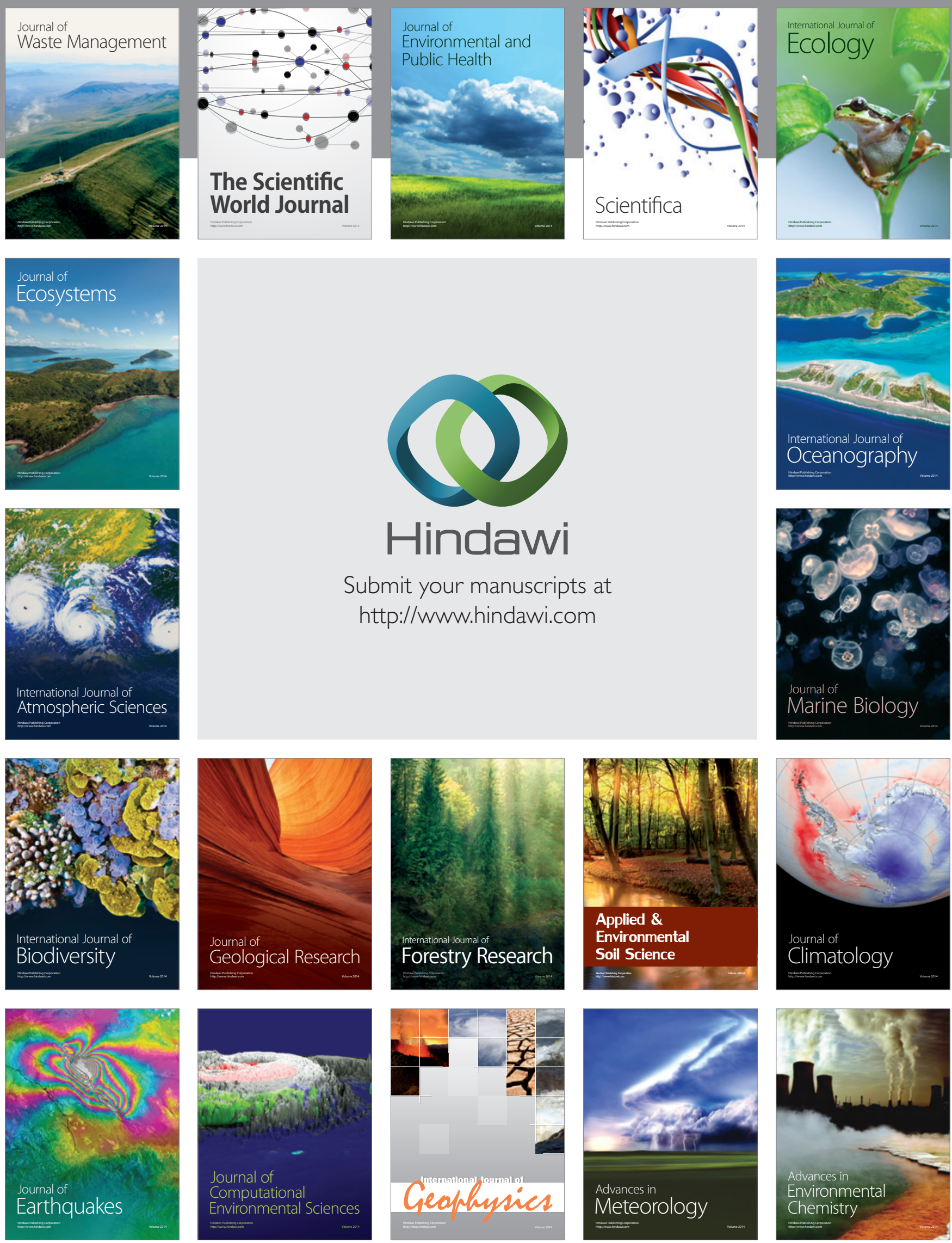